and transection of those vessels was very important,because thyroid tissue removal between the trachea and RLN became more convenient. This technique guaranteed safety of the RLN.Our technique of thyroidectomy allowed to find and save $15(0.37 \%)$ nonrecurrent laryngeal nerves among 4070 thyroid patients. Postoperative laryngoscopical control allowed estimating the postoperative laryngeal palsy rate objectively. In 21 cases RLN was restored with the perineural suturing under the IONIMcontrol. During 1-2 years postoperatively 15 (71.4\%) demonstrated vocal cord function improvement.To protect SAN during lateral neck dissection the procedure of its surgical separation was specified. The best place for the first nerve visualization (because of safety) was the level of sternocleidomastoid muscle's upper third where it accompanied the internal jugular vein. Our technique assumes dissection of SAN from above with separating and saving of nervous branches to sternocleidomastoid muscle's, C3 and TM with consecutive moving of SAN from inner to the outer part of the sternocleidomastoid muscle's by the rubber holder and forceps.

Conclusion As a result of regular use of suggested RLN, SAN dissection and IONIMtechnique, the postoperative morbidity rate decreased significantly.

\section{OC55 INHIBITORS IN ALBANIAN CHILDREN WITH HEMOPHILIA A}

Arlind Deveja*, Donjeta Bali, Mirela Xhafa, Mirzana Kapllanaj, Etleva Refatllari, Anila Godo. University Hospital Center 'Mother Teresa', Tirana, Albania

\subsection{6/archdischild-2019-epa.52}

Objective To identify the data related with therapeutic and patients characteristics and their correlation with the inhibitor's presence in hemophilia A in Pediatric Hematologic/Oncologic Unit. University Hospital Centre 'Mother Teresa', Tirana, Albania, the unique national service that treat pediatric coagulopathies.

Design This analytic, observational, retrospective and case control study analyses 32 patients (0-14 years old) diagnosed and treated in our center for hemophilia $A$, that had been tested for inhibitor presence (32 from 45 children with hemophilia A in Albania).

Outcome measures Inhibitor's presence (qualitative test) after 50 days of exposure, severity of the disease, breastfeeding, family history for hemophilia, age and purpose of first FVIII exposure, age of $50^{\text {th }}$ exposure, time between first and $50^{\text {th }}$ exposure, surgery up to $50^{\text {th }}$ day of exposure, type and number of exposures episode (minor or major) and FVIII average dosage.

Results Inhibitors were present in 13 from 32 patients. The mean age of the first exposure to FVIII is 20.1 months old and in 25 of 32 patients hemorrhage was the reason for the first exposure. There was no significant statistical difference between inhibitors presence and age and reasons of first exposure. Neither nor the age of the $50^{\text {th }}$ exposure wasn't correlated with inhibitors presence. The mean age between first and $50^{\text {th }}$ day of exposure was 24.2 months and there was a positive correlation between this data and inhibitors presence $(\mathrm{t}$-Test $=2.267 ; \mathrm{df}=30 ; \mathrm{p}=0.031)$, also the number of episodes of exposition until the $50^{\text {th }}$ day of exposition (avg.6.5) was correlated with inhibitors presence ( $\mathrm{t}$-Test $=$ $3.270 ; \mathrm{df}=30 ; \mathrm{p}=0.003)$. We had more major exposure episodes $(78.1 \%)$ and this data influenced in inhibitor's presence (Fisher's Test $=6.131$; $\mathrm{df}=1 ; \mathrm{p}=0.015$ ). The average dosage of FVIII was also correlated with inhibitor's presence $\left(\chi^{2}=12,799 ; \mathrm{df}=2 ; \mathrm{p}=0.002\right)$. Severity of the disease and family history for hemophilia weren't correlated with the inhibitors. Breastfeeding with associated with lower risk for inhibitors presence $(t=7.166 ; \mathrm{df}=; \mathrm{p}=0.011)$.

Conslusions Inhibitor's presence percentage in Albanian pediatric patients with hemophilia $\mathrm{A}$ is $40.6 \%$. This data is much higher than the literature reports. Factors influencing more in the development or presence of inhibitors in our population are: high dosage of factor VIII, the high frequency of exposure episodes and the short time between first and the $50^{\text {th }}$ exposure day.

\section{OC56 HYPERTHERMIC INTRAPERITONEAL CHEMOTHERAPY FOR TREATMENT OF DESMOPLASTIC SMALL ROUND CELL TUMOURS IN A CHILD; A U.K. FIRST} ${ }^{1,2}$ Peter Donnelly*, ${ }^{1}$ Kestner Sam, ${ }^{1}$ Katy Robinson, 'Sucheta Vaidya, 'Bruce Okoye,
${ }^{1}$ Linda Murdoch, 'Buvana Dwarakanathan. 'St George's Hospial, London, UK; ' ${ }^{1}$ Evelina
London Children's Hospital, London, UK

\subsection{6/archdischild-2019-epa.53}

Introduction Desmoplastic small round cell tumours are rare, aggressive neoplastic tumours with a predisposition to affect the intra-abdominal cavity.

Hyperthermic intraperitoneal chemotherapy (HIPEC) is a mode of delivering concentrated, heated chemotherapy directly into the abdominal cavity. This has been used previously in adult patients and we describe the first UK case of HIPEC for a desmoplastic small round cell tumour in a child.

Case summary An 8 year old girl (AB) was diagnosed with desmoplastic small round cell tumours after presenting with a ten day history of constipation and abdominal discomfort. Initial assessment revealed a large palpable abdominal mass. An ultrasound demonstrated a large heterogenous mass with central cystic necrosis. Two additional lesions were noted within the liver in addition to a $22 \mathrm{~mm}$ omental deposit. Bone marrow aspirates and biopsies confirmed the diagnosis.

$\mathrm{AB}$ had eleven rounds of chemotherapy in total with the most recent cycle completing in April 2018. A follow-up MRI showed a residual pelvic tumour of $3.1 \mathrm{~cm}$ indenting the superior wall of the bladder.

After MDT discussion the decision was made to proceed with macroscopic resection of tumours followed by cisplatin HIPEC. Intraoperatively, lesions were noted in the following sites: omentum; pouch of Douglas; left ovary and fimbrae; jejunum and diaphragm. After resection was completed, cisplatin heated to 41 degrees $\mathrm{C}$ was washed over the peritoneum. This was tolerated with noradrenaline used to optimise haemodynamics. Postoperatively she was admitted to PICU for monitoring and observation.

During PICU admission AB remained clinically stable. Active interventions included GCSF, thoracic epidural and PCA for analgesia, electrolyte optimisation and hyperhydration. $\mathrm{AB}$ was ready for discharge from PICU after 4 days.

Conclusion We described the first case of HIPEC being used in paediatrics in the UK. This technique has been well tolerated and could be considered in other cases of intra-abdominal neoplasia with metastases. 